\title{
CHARACTERIZING TRANSLATIONS ON GROUPS BY COSETS OF THEIR SUBGROUPS
}

\author{
GÁBOR HORVÁTH, PETER MAYR, AND ANDRÁS PONGRÁCZ
}

\begin{abstract}
On a group, constant functions and left translations by group elements map left cosets into left cosets for every subgroup. We determine classes of groups for which this property of preserving cosets characterizes constants and translations, e.g., finite non-abelian groups that are perfect, partitioned, primitive, or generated by elements of prime order $p$. For certain classes of groups we construct other coset-preserving functions, in particular, power endomorphisms and functions defined in terms of the subgroup lattice.
\end{abstract}

Address (1): Institute of Mathematics, University of Debrecen, Pf. 12, Debrecen, 4010, Hungary

E-mail address: ghorvath@math.unideb.hu

Address (2): CAUL, Av. Prof. Gama Pinto 2, 1649-003 Lisboa, Portugal \& Institut für Algebra, JKU Linz, Altenberger Strasse 69, 4040 Linz, Austria

E-mail address: stein@cii.fc.ul.pt

Address (3): Department of Algebra and Number Theory, Eötvös Loránd University, 1117 Budapest, Pázmány Péter sétány 1/C, Hungary

E-mail address: pongeee@cs.elte.hu

Running title: Translations on Groups

\section{INTRODUCTION}

In this paper we investigate interactions of the structure of a group with the structure of its subgroup lattice. It is a longstanding problem whether every finite lattice is isomorphic to an interval in the subgroup lattice for some finite group. Various approaches, e.g., by Baddeley and Lucchini [2] and most recently by Aschbacher [1], were aimed at showing that the answer is negative. Still the problem remains open.

Date: February 3, 2011.

2000 Mathematics Subject Classification. 20D30 (06B05,08A40).

Key words and phrases. translations on groups, coset preserving functions, perfect groups, primitive groups, partitioned groups, subgroup lattices. 
The question originates from general algebraic structures by the following result of Pálfy and Pudlák [10]: if all finite lattices are representable as congruence lattices of finite algebras, then all finite lattices occur as intervals in subgroup lattices of finite groups (equivalently, as congruence lattices of finite $G$-sets). Recall that for a permutation group $G$ on a set $\Omega$, the algebra $\langle\Omega, G\rangle$ is called a $G$-set.

The result of Pálfy and Pudlák does not mean that every representable finite lattice is the congruence lattice of a $G$-set. However for certain lattices this stronger implication is true. In [13] Snow observed that if a finite order polynomially complete lattice is isomorphic to a congruence lattice of a finite algebra, then it is isomorphic to the congruence lattice of a finite vector space or of a finite affine complete $G$-set (see [7] for various concepts of completeness and [8] for a general introduction to Universal Algebra). Here, for $k \in \mathbb{N}$, an algebra $\mathbf{A}$ is called $k$-affine complete if every $k$-ary congruence preserving function on the universe of $\mathbf{A}$ is a polynomial; $\mathbf{A}$ is affine complete if it is $k$-affine complete for all $k \in \mathbb{N}$. In this paper we start the investigation of affine complete $G$-sets. Our goal is to obtain some qualitative understanding of how common they are and to obtain information on the structure of their congruence lattices.

From Pálfy's classification of minimal algebras [9] we obtain:

Theorem 1. Let $G$ be a finite non-trivial permutation group on a set $\Omega$. Assume that $\langle\Omega, G\rangle$ is 1-affine complete. Then

(1) $\langle\Omega, G\rangle$ is affine complete or

(2) $|\Omega|=2$ or

(3) there exists a vector space $V$ over some field $F$ such that $\langle\Omega, G\rangle$ is isomorphic to

$$
\langle V,\{V \rightarrow V, x \mapsto f x+v \mid f \in F, f \neq 0, v \in V\}\rangle .
$$

By Theorem 1 we may restrict ourselves to the investigation of 1affine complete $G$-sets. In this paper we will consider $G$-sets with the regular action, i.e., $\Omega=G$. Therefore we set the following definitions.

For a group $G$ we call a function $f: G \rightarrow G$ compatible if for every subgroup $H \leq G$, and for every elements $x, y \in G$ from the same left coset of $H$ (i.e., $x H=y H$ ) we have that $f(x)$ and $f(y)$ are in the same left coset of $H$ (i.e., $f(x) H=f(y) H)$. Let us note that for every $g \in G$

- the constant function, $G \rightarrow G, x \mapsto g$,

- the left translation, $G \rightarrow G, x \mapsto g x$,

are compatible on $G$.

If every compatible function $f: G \rightarrow G$ is either constant or a left translation, then we say $G$ is translation complete or $t$-complete 
for short. It is easy to see that the compatible functions on $G$ are exactly the unary congruence preserving functions on the regular $G$ set; moreover $G$ is t-complete if and only if the regular $G$-set is 1-affine complete. Nevertheless one can consider t-completeness as a notion in pure group theory, and we use this perspective for the remainder of the paper. Our main reference for definitions and standard results on group theory is [11].

The more subgroups a group has, the more restrictions a compatible function has to satisfy. Hence groups with 'many' subgroups should have 'few' compatible functions apart from constants and translations. In fact we prove the following in Section 4.

Theorem 2. All of the following groups are t-complete:

(1) groups generated by involutions,

(2) finite groups all of whose minimal normal subgroups are nonabelian,

(3) the free group on 2 or more generators,

(4) finite perfect groups,

(5) linear groups $G$ with $\operatorname{SL}(n, F) \leq G \leq \mathrm{GL}(n, F)$ for a field $F$ and $n>1$,

(6) finite semidirect products $A \rtimes B$ where $A$ is elementary abelian and $B$ acts as non-trivial group of automorphisms on $A$,

(7) finite non-abelian primitive permutation groups,

(8) finite non-abelian groups that have a trivial Frattini subgroup and do not split into a direct product of non-trivial subgroups of coprime order,

(9) finite non-abelian groups that are generated by elements of prime order and do not split into a direct product of non-trivial subgroups of coprime order,

(10) finite non-abelian partitioned groups.

From this theorem it seems that t-complete groups are quite abundant. By (1) all Coxeter groups are t-complete, in particular elementary abelian 2-groups, generalized dihedral groups, and all symmetric groups $S_{n}$ for $n \in \mathbb{N}$ are t-complete. Alternating groups $A_{n}$ for $n \geq 4$ are t-complete by (7). Every finite non-abelian simple group is t-complete by (2). For every field $F$ and $n>1$ we have that $\operatorname{PSL}(n, F)$ is t-complete $\left(\operatorname{PSL}\left(2, \mathbb{Z}_{2}\right) \cong S_{3}, \operatorname{PSL}\left(2, \mathbb{Z}_{3}\right) \cong A_{4}\right.$, otherwise $\operatorname{PSL}(n, F)$ is simple and generated by involutions). By (9) all finite non-abelian groups of exponent $p$ are t-complete. Frobenius groups are t-complete by $(10)$.

In Section 4.1 we present some expanding properties for t-complete groups. Items (1) and (2) of Theorem 2 are proved in Section 4.2. 
Items (3), (4), and (5) are shown in Section 4.3. In Section 4.4 we investigate basic properties of compatible functions on partitioned groups. In Section 4.5 we investigate semidirect products and prove items (6) and (7). Theorem 36 generalizes item (8). In Section 4.6 we prove Theorem 38, which generalizes item (9). Then in Section 4.7 we revisit partitioned groups and prove item (10). We finish our discussion by investigating maximal t-complete quotients of a group in Section 4.8 and maximal t-complete subgroups of a group in Section 4.9 .

To show that a group $G$ is not t-complete we have to find a non-trivial compatible function on it. First we investigate some general properties of compatible functions in Section 2. Then we consider power functions and construct functions for groups with particular subgroup lattices in Section 3. Thus we obtain the following.

Theorem 3. None of the following groups are t-complete:

(1) abelian groups of exponent greater than 2,

(2) Hamiltonian groups,

(3) powerful p-groups of exponent greater than 2,

(4) non-abelian groups, whose index of the center is finite and not divisible by the exponent of the group,

(5) groups $G$ with proper non-trivial subgroups $A, B$ such that every subgroup of $G$ contains $A$ or is contained in $B$,

(6) direct products of two non-trivial coprime torsion groups.

Hence the smallest non-abelian group that is not t-complete is the quaternion group with 8 elements.

Items (1), (2), (3), and (4) of Theorem 3 are proved in Section 3.1. We show (5) and (6) in Section 3.2.

The subgroups of a group $G$ form a lattice $\operatorname{Sub}(G)$ with the operations $A \wedge B=A \cap B$ and $A \vee B=\langle A \cup B\rangle$. In Section 3.2 we investigate connections between the properties of this lattice and t-completeness. Recall that a group with distributive subgroup lattice is locally cyclic by a result of Ore [12, Theorem 1.2.3]. Thus by item (1) of Theorem 2 and item (1) of Theorem $3, \mathbb{Z}_{2}$ is the only non-trivial t-complete group with distributive subgroup lattice.

In general, whether or not a group is t-complete is not determined by its subgroup lattice alone. The subgroup lattices of $S_{3}$ and $\left(\mathbb{Z}_{3}\right)^{2}$ are isomorphic but $S_{3}$ is t-complete by item (1) of Theorem 2 while $\left(\mathbb{Z}_{3}\right)^{2}$ is not t-complete by item (1) of Theorem 3. Still, for non-abelian groups that are generated by their minimal subgroups or that have 
trivial Frattini subgroup we obtain the following characterization of tcompleteness in terms of subgroup lattices from items (8) and (9) of Theorem 2 and item (6) of Theorem 3.

Corollary 4. Let $G$ be a finite non-abelian group. Assume that the join of all atoms in $\operatorname{Sub}(G)$ is $G$ or that the intersection of all coatoms in $\operatorname{Sub}(G)$ is 1. Then $G$ is t-complete if and only if $\operatorname{Sub}(G)$ is not a direct product of two non-trivial lattices.

Moreover, in Proposition 42 we observe that neither 't-completeness' nor 'not t-completeness' is hereditary to subgroups, to normal subgroups, or to factor groups. We finish the paper by mentioning some open problems in Section 5.

\section{Compatible FunCtions}

Let us start by examining compatible functions. We present some basic lemmas which are used later on in the paper. The first lemma of the section tells us that for checking whether or not a group is t-complete, we only need to consider compatible functions fixing the identity element of the group:

Lemma 5. A group $G$ is t-complete if and only if every compatible function $f$ on $G$ with $f(1)=1$ satisfies either $f(G)=1$ or $f=\operatorname{id}_{G}$.

Proof. The 'only if' direction is immediate: if $f$ is constant and $f(1)=$ 1 then $f(G)=1$; if $f$ is a translation and $f(1)=1$ then $f=\mathrm{id}_{G}$.

For the 'if' direction, let $h$ be a compatible function on $G$. Then $f$ : $G \rightarrow G, x \mapsto h(1)^{-1} h(x)$, is compatible as well and satisfies $f(1)=1$. If $f(G)=1$, then $h$ is constant; if $f=\mathrm{id}_{G}$, then $h$ is a translation by $h(1)$.

From now on we mostly consider compatible functions fixing the identity. By the following two lemmas compatibility is inherited by subgroups and factor groups.

Lemma 6. Let $f$ be compatible on a group $G$ with $f(1)=1$, and let $H \leq G$. Then $f(H) \subseteq H$, and the restriction $\left.f\right|_{H}$ is compatible on $H$.

Proof. Straightforward.

Lemma 7. Let $G$ be a group with normal subgroup $N$, and let $f$ be a compatible function on $G$. Then

$$
f_{N}: G / N \rightarrow G / N, x N \mapsto f(x) N,
$$

is compatible on $G / N$.

Proof. Straightforward. 
We note that in general not every compatible function on a quotient $G / N$ is induced by a compatible function on $G$. Moreover, not every compatible function on a subgroup $H$ is the restriction of a compatible function on $G$. For example, every compatible function on $\left(\mathbb{Z}_{3}\right)^{2}$ is of the form $x \mapsto g+k x$ for some $g \in\left(\mathbb{Z}_{3}\right)^{2}$ and some $k \in\{0,1,2\}$ as we later see in Lemma 13 . However on $\mathbb{Z}_{3}$ every function is compatible.

On products we can study the compatible functions componentwise:

Lemma 8. Let $G$ be a semidirect product of a normal subgroup $A$ and a complement $B$. Let $f$ be a compatible function on $G$ with $f(1)=1$. Then $f(a b)=f(a) f(b)$ for all $a \in A, b \in B$.

Proof. Let $a \in A, b \in B$. Then $a b B=a B$ yields $f(a b) B=f(a) B$. Since $A$ is normal, we have $a b A=a A b=A b=b A$. Hence $f(a b) A=$ $f(b) A=A f(b)$. Thus

$$
f(a b) \in f(a) B \cap A f(b) .
$$

Since $G$ is a semidirect product, every intersection of an $A$-coset and a $B$-coset contains exactly 1 element. Since $f(a) f(b) \in f(a) B \cap A f(b)$ by Lemma 6 , we obtain $f(a) B \cap A f(b)=\{f(a) f(b)\}$ and the assertion follows.

\section{Groups that are not T-COMplete}

In this section we prove Theorem 3 by investigating properties that prevent groups from being t-complete.

3.1. Power maps. On a group $G$ a power map is a function $f: G \rightarrow$ $G, x \mapsto x^{k}$, for some $k \in \mathbb{Z}$. Note that every subgroup of $G$ is invariant under such functions and that $f(1)=1$ holds. Power maps are not always compatible, e.g., for $G=S_{3}, H=\langle(12)\rangle, x=(13), y=(123)$ we have $x H=y H$ but $x^{2} H \neq y^{2} H$. Nevertheless power maps preserve cosets of normal subgroups by the following auxiliary result.

Lemma 9. Let $G$ be a group, let $k \in \mathbb{Z}$, and let $f: G \rightarrow G, x \mapsto x^{k}$.

(1) If $N$ is a normal subgroup of $G$, then $x N=y N$ implies $f(x) N=f(y) N$ for all $x, y \in G$.

(2) If $f$ is a homomorphism, then $f$ is compatible.

Proof. Assertion (1) is immediate in the factor group $G / N$.

For proving (2), let $H \leq G$, and let $x, y \in G$ be such that $x H=y H$. Then $y^{-1} x \in H$ implies $\left(y^{-1} x\right)^{k} \in H$. Since $\left(y^{-1} x\right)^{k}=y^{-k} x^{k}$ by the homomorphism property, we have $y^{-k} x^{k} \in H$. Thus $f(x) H=x^{k} H=$ $y^{k} H=f(y) H$. 
By (1) of the previous lemma power maps on abelian groups are compatible. In fact we have the following.

Corollary 10. Let $G$ be a t-complete group in which every subgroup is normal. Then $x^{2}=1$ for all $x \in G$, and consequently $G$ is an elementary abelian 2-group.

Proof. By (1) of Lemma 9 the function $f: G \rightarrow G, x \mapsto x^{2}$, is compatible on $G$. Since $f(1)=1$, either $f$ is constant or the identity on $G$. Hence $f(G)=1$ or $G=1$, which proves the result.

Non-abelian groups in which every subgroup is normal are called Hamiltonian groups. Thus we have proved items (1) and (2) of Theorem 3. Later we see that elementary abelian 2-groups are t-complete, as these groups are generated by involutions (Corollary 21). This completes the characterization of t-complete abelian and Hamiltonian groups.

We thank the referee for calling our attention to the notion of powerful groups, which allowed us to obtain item (3) of Theorem 3. Let $p$ be a prime. A finite $p$-group is powerful if $p$ is odd and $G^{\prime} \subseteq\left\langle x^{p} \mid x \in G\right\rangle$ or if $p=2$ and $G^{\prime} \subseteq\left\langle x^{4} \mid x \in G\right\rangle$.

Corollary 11. Let $G$ be a t-complete finite powerful p-group. Then $G$ is an elementary abelian 2-group.

Proof. Assume $\exp G=p^{e}$ for $e \in \mathbb{N}$. By [3, p. 45, Exercise 2.5] the function $f: G \rightarrow G, x \mapsto x^{p^{e-1}}$, is an endomorphism. Then $f$ is compatible on $G$ by (2) of Lemma 9 . Since $f(1)=1$ and $f(G) \neq 1$, $f$ is the identity on $G$. Hence $\exp G=p$ and $G^{\prime}=1$. As an abelian t-complete group, $G$ is an elementary abelian 2-group by Corollary 10 .

As example we mention that for an odd prime $p$ every finite metacyclic $p$-group $G$ with a cylic normal subgroup $N$ and cyclic quotient $G / N$ is powerful (note that $G / N^{p}$ is abelian). Hence by Corollary 11 none of these groups are t-complete.

Let us recall, that the transfer to the center of a non-abelian group is always a power endomorphism [11, 10.1.3]. Combining this fact with (2) of Lemma 9, we obtain that t-complete groups have 'small' centers proving item (4) of Theorem 3:

Corollary 12. Let $G$ be a non-abelian t-complete group whose center $Z(G)$ has finite index in $G$. Then the exponent of $G$ divides $|G: Z(G)|$.

Proof. The transfer into the center is a power endomorphism of the form $t: G \rightarrow G, x \mapsto x^{|G: Z(G)|}$, by $[11,10.1 .3]$. Then $t$ is compatible on 
$G$ by Lemma 9 . As $G \neq Z(G)$, we have $t \neq \operatorname{id}_{G}$, thus $t(G)=1$. Hence $\exp G$ divides $|G: Z(G)|$.

The abelian groups $G$ for which all compatible functions are of the form $G \rightarrow G, x \mapsto g x^{k}$, for some $g \in G, k \in \mathbb{Z}$ were determined by Kaarli in [6]. These are exactly the 1-affine complete abelian groups. For the convenience of the reader we give the proof of the following elementary fact which we use when considering direct products in Section 4.1 and groups generated by elements of order $p$ in Section 4.2.

Lemma 13. Let $A, B$ be isomorphic cyclic groups. Then every compatible function $f$ on $A \times B$ with $f(1)=1$ is a power map.

Proof. Let $a, b$ be generators of $A, B$, respectively. Let $G=A \times B$. Let $f$ be a compatible function $G$ with $f(1)=1$, and let $y \in B$. By Lemma 6 there exist integers $k, l$ such that $f(a)=a^{k}, f(y)=y^{l}$. Then Lemma 8 implies $f(a y)=a^{k} y^{l}$. First assume $|A|=|B|=n$ for some $n \in \mathbb{N}$. Since $f(a y)$ is in the subgroup $\langle a y\rangle$, we obtain $l \equiv k$ $(\bmod \operatorname{ord}(y))$. If $A, B$ are infinite cyclic groups, then $f(a y) \in\langle a y\rangle$ yields $l=k$. In both cases we have $f(y)=y^{k}$ for all $y \in B$, in particular $f(b)=b^{k}$.

By switching coordinates the same argument now yields $f(x)=x^{k}$ for all $x \in A$. For $x \in A, y \in B$, Lemma 8 then implies $f(x y)=$ $f(x) f(y)=x^{k} y^{k}=(x y)^{k}$. Hence $f$ is the $k$-th power map on $G$.

3.2. Subgroup lattice. In this section we determine certain lattices that cannot be isomorphic to subgroup lattices of t-complete groups. The following result, which proves item (5) of Theorem 3, is due to E. Aichinger (private communication).

Proposition 14. Let $G$ be a group with proper, non-trivial subgroups $A, B$ such that for all $H \leq G$ either $A \leq H$ or $H \leq B$. Let $a \in A$ be an element such that $a \neq 1$. Then

(1) the function

$$
f: G \rightarrow G, x \mapsto \begin{cases}1, & \text { if } x \in B, \\ a, & \text { else },\end{cases}
$$

is compatible, and

(2) the group $G$ is not t-complete.

Proof. Let $H \leq G$, and let $x, y \in G$ be such that $x H=y H$. If $A \leq H$, then $f(G) \subseteq A$ yields $f(x) H=H=f(y) H$. If $H \leq B$, then $x H=y H$ yields $x B=y B$ and $f(x)=f(y)$. Hence $f$ is compatible.

Since $B$ is non-trivial, the function $f$ is neither constant nor a translation, which proves the second assertion. 
We finish Section 3.2 with an observation about the case when the subgroup lattice of a group $G$ is a direct product of two nontrivial lattices. We say that torsion groups $A, B$ are coprime if $\operatorname{gcd}(\operatorname{ord}(a), \operatorname{ord}(b))=1$ for all $a \in A, b \in B$. Groups whose subgroup lattices are direct products of two lattices were characterized as direct products of coprime torsion groups in [14]. By the next result they have non-trivial compatible functions as well.

Proposition 15. The following are equivalent for a group $G$ :

(1) The subgroup lattice of $G$ is a direct product of two non-trivial lattices.

(2) $G$ is a direct product of non-trivial coprime torsion groups $A$ and $B$.

(3) $G$ is generated by proper, non-trivial subgroups $A, B$, and there exists a compatible function $f$ on $G$ such that $f(A)=1$ and $\left.f\right|_{B}=\operatorname{id}_{B}$.

Proof. $(1) \Leftrightarrow(2)$ is [14, Lemma 3] (or see [12, Lemma 1.6.4, Theorem 1.6.5]).

$(2) \Rightarrow(3)$ : Let $A, B$ be torsion groups such that $\operatorname{gcd}(\operatorname{ord}(a), \operatorname{ord}(b))=$ 1 for all $a \in A, b \in B$. For every $H \leq A \times B$ there exist $C \leq A$ and $D \leq B$ such that $H=C \times D$ by [12, Lemma 1.6.4]. It is easy to check that

$$
f: A \times B \rightarrow A \times B,(x, y) \mapsto\left(1_{A}, y\right),
$$

is compatible on $A \times B$. Indeed, let $\left(a_{1}, b_{1}\right) H=\left(a_{2}, b_{2}\right) H$. Then $b_{1} D=b_{2} D$ and consequently $f\left(\left(a_{1}, b_{1}\right)\right) H=\left(1, b_{1}\right) H=\left(1, b_{2}\right) H=$ $f\left(\left(a_{2}, b_{2}\right)\right) H$.

$(3) \Rightarrow(2)$ : Let $1<A, B<G$, and let $f: G \rightarrow G$ be a compatible function such that $f(A)=1,\left.f\right|_{B}=\operatorname{id}_{B}$. Let $a \in A, a \neq 1$, and $b \in B, b \neq 1$. Since $b\left\langle b^{-1} a\right\rangle=a\left\langle b^{-1} a\right\rangle$, we have $b\left\langle b^{-1} a\right\rangle=f(b)\left\langle b^{-1} a\right\rangle=$ $f(a)\left\langle b^{-1} a\right\rangle=\left\langle b^{-1} a\right\rangle$. Hence $b \in\left\langle b^{-1} a\right\rangle$ and consequently $a=b \cdot b^{-1} a \in$ $\left\langle b^{-1} a\right\rangle$. Since $a, b$ are both in the cyclic group generated by $b^{-1} a$, they commute. Then $\langle a\rangle\langle b\rangle$ is cyclic with $\langle a\rangle \cap\langle b\rangle=1$ since $f(A)=1$, $\left.f\right|_{B}=\operatorname{id}_{B}$. Now $\left\langle b^{-1} a\right\rangle$ cannot be infinite, as any two non-trivial subgroups of the group $\mathbb{Z}$ meet non-trivially. Hence $\left\langle b^{-1} a\right\rangle$ is finite, and the orders of $a$ and $b$ are finite and coprime.

Proposition 15 immediately implies (6) of Theorem 3. Thus the proof of Theorem 3 is complete.

\section{T-COMPLETE GROUPS}

In this section we present some examples for t-complete groups and prove Theorem 2 . 
4.1. Expanding properties. First we investigate how one can construct t-complete groups from smaller t-complete groups. Let us start by checking direct products.

Proposition 16. Let $A, B$ be groups of finite exponents such that $\exp (A)$ divides $\exp (B)$.

(1) Let $f$ be a compatible function on $A \times B$ with $f(1)=1$. Then there exists $k \in \mathbb{Z}$ such that for all $x \in A \times B$ whose order divides $\exp (A)$ we have $f(x)=x^{k}$.

(2) If $B$ is t-complete, then $A \times B$ is t-complete.

Proof. First we prove (1). Let $G=A \times B$, and let $f$ be a compatible function on $G$ with $f(1)=1$. Let $q$ be a maximal prime power dividing $\exp (A)$. Let $a \in A, b \in B$ be of order $q$ : $\operatorname{ord}(b)=\operatorname{ord}(a)=q$. Consider the subgroup $\langle a, b\rangle$. Now $a$ and $b$ commute, and both $a$ and $b$ generate a cyclic group of order $q$. By Lemma 13 there exists an integer $k_{q}$, such that $f(x)=x^{k_{q}}$ for every $x \in\langle a, b\rangle$.

Let $c \in A$ such that ord $(c)$ divides $q$. We claim that

$$
f(c)=c^{k_{q}} .
$$

Note that $c$ and $b^{\operatorname{ord}(b) / \operatorname{ord}(c)}$ commute and both generate a cyclic group of order $\operatorname{ord}(c)$. Thus by Lemma 13, $f$ is a power map on $\left\langle c, b^{\operatorname{ord}(b) / \operatorname{ord}(c)}\right\rangle$. Since $f\left(b^{\operatorname{ord}(b) / \operatorname{ord}(c)}\right)=b^{k_{q} \operatorname{ord}(b) / \operatorname{ord}(c)}$, we have $f(x)=$ $x^{k_{q}}$ for all $x \in\left\langle c, b^{\operatorname{ord}(b) / \operatorname{ord}(c)}\right\rangle$. Hence (4.1) is proved.

Now let $d \in B$ such that $\operatorname{ord}(d)$ divides $q$. We consider the subgroup generated by $d$ and $a^{\operatorname{ord}(a) / \operatorname{ord}(d)}$. Then the same argument as above yields that $f(d)=d^{k_{q}}$.

From Lemma 8 we can conclude that $f(x)=x^{k_{q}}$ for every $x \in G$, whose order divides $q$. By the Chinese Remainder Theorem, we have $k \in \mathbb{Z}$ such that $k \equiv k_{q}(\bmod q)$ for every maximal prime power $q$ that divides $\exp (A)$. Finally let $y \in G$ such that $\operatorname{ord}(y)$ divides $\exp (A)$. Then $y$ is a product of elements of prime power order, which are mapped to their $k$-th power by $f$. By Lemma 8 we obtain $f(y)=y^{k}$.

For (2) let $f$ be a compatible function on $A \times B$ with $f(1)=1$. Thus $f$ is compatible on $B$ by Lemma 7 , and we have either $f(B)=1$ or $\left.f\right|_{B}=\operatorname{id}_{B}$. By (1) we have $f(A)=1$ if $f(B)=1$, or we have $\left.f\right|_{A}=\operatorname{id}_{A}$ if $\left.f\right|_{B}=\operatorname{id}_{B}$. Now the result follows from Lemma 8 .

We continue with showing that t-completeness can extend from a subgroup under certain circumstances. We use Lemma 17 for example to prove that non-abelian linear groups and non-abelian primitive permutation groups are t-complete. 
Lemma 17. Let $G$ be a group, let $N \leq G$, and let $\mathcal{H}=\{H \leq$ $G \mid N H=G\}$. Assume that $\bigcap \mathcal{H}=1$ and that every compatible function $f$ on $G$ with $f(1)=1$ satisfies either $f(N)=1$ or $\left.f\right|_{N}=\operatorname{id}_{N}$. Then $G$ is t-complete.

Proof. Let $f$ be a compatible function on $G$ with $f(1)=1$. For $x \in G$ and $H \in \mathcal{H}$, we have a suitable $h \in H$ such that $x h \in N$. Since $x H=x h H$, we get

$$
f(x) H=f(x h) H .
$$

If $f(N)=1$, then $f(x) \in H$ by (4.2). Hence $f(x) \in \bigcap \mathcal{H}=1$ and $f(G)=1$. If $\left.f\right|_{N}=\operatorname{id}_{N}$, then $f(x h)=x h$ yields $f(x) \in x H$ by (4.2). Hence $f(x) \in x \bigcap \mathcal{H}=\{x\}$ and $f=\operatorname{id}_{G}$.

4.2. Generating by subgroups. We continue our investigation with the situation when t-complete subgroups generate a group. The main result in this section is Theorem 20, saying that a group generated by tcomplete subgroups is t-complete unless it is a direct product of coprime torsion groups. This fact has many corollaries, such as items (1) and (2) of Theorem 2.

First we prove two lemmas about the connection of subgroups and compatible functions.

Lemma 18. Let $G$ be a group, let $A \leq G$, such that for every compatible function $h$ on $G$ with $h(1)=1$ either $h(A)=1$ or $\left.h\right|_{A}=\operatorname{id}_{A}$. Let $f$ be a compatible function on $G$, and let $g \in G$. Then either

(1) $f(g A)=f(g)$ or

(2) $f(g a)=f(g)$ a for all $a \in A$.

Proof. As a composition of compatible functions, $h: G \rightarrow G, x \mapsto$ $f(g)^{-1} f(g x)$, is compatible on $G$ with $h(1)=1$. If $h(A)=1$, then $f(g A)=f(g)$. If $h(a)=a$ for every $a \in A$, then $f(g a)=f(g) a$.

Lemma 19. Let $G$ be a group, and let $\mathcal{H}$ be a set of subgroups of $G$ such that $\langle\bigcup \mathcal{H}\rangle=G$. Assume that for every compatible function $f$ on $G$ with $f(1)=1$ either $f\left(A^{g}\right)=1$ for all $A \in \mathcal{H}$ and $g \in G$ or $\left.f\right|_{A^{g}}=\operatorname{id}_{A^{g}}$ for all $A \in \mathcal{H}$ and $g \in G$. Then $G$ is t-complete

Proof. Let $f$ be a compatible function on $G$ with $f(1)=1$. First assume that $f\left(A^{g}\right)=1$ for every $A \in \mathcal{H}$ and every $g \in G$. We prove that

$$
\forall n \in \mathbb{N} \quad \forall u_{1}, \ldots, u_{n} \in \bigcup \mathcal{H}: f\left(u_{1} \cdots u_{n}\right)=1 .
$$

Seeking a contradiction let us suppose that we have a minimal $n \in$ $\mathbb{N}$ with $u_{1}, \ldots, u_{n} \in \bigcup \mathcal{H}$ such that $f\left(u_{1} \cdots u_{n}\right) \neq 1$. Then $n>1$. 
By Lemma 18 we have $f\left(u_{1} \cdots u_{n}\right) \in\left\{f\left(u_{1} \cdots u_{n-1}\right), f\left(u_{1} \cdots u_{n-1}\right) u_{n}\right\}$. Since $f\left(u_{1} \cdots u_{n-1}\right)=1$ by the minimality of $n$, this yields

$$
f\left(u_{1} \cdots u_{n}\right)=u_{n} .
$$

Since $f\left(A^{u_{n}}\right)=1$ for all $A \in \mathcal{H}$, we obtain similarly that

$$
f\left(u_{1} \cdots u_{n}\right)=f\left(u_{1} \cdots u_{n-2} u_{n} u_{n-1}^{u_{n}}\right)=u_{n-1}{ }^{u_{n}} .
$$

Then $f\left(u_{1} \cdots u_{n}\right)=u_{n}=u_{n-1} u_{n}$ implies $u_{n}=u_{n-1}$. Let $v=u_{n-1}{ }^{2}$. Then $v$ is in $\bigcup \mathcal{H}$. Now $f\left(u_{1} \cdots u_{n}\right)=f\left(u_{1} \cdots u_{n-2} v\right)=1$ by the minimality of $n$. This contradiction proves (4.3).

Next we assume that $\left.f\right|_{A^{g}}=\operatorname{id}_{A^{g}}$ for every $A \in \mathcal{H}$ and every $g \in G$. We prove that

$$
\forall n \in \mathbb{N} \quad \forall u_{1}, \ldots, u_{n} \in \bigcup \mathcal{H}: f\left(u_{1} \cdots u_{n}\right)=u_{1} \cdots u_{n} .
$$

Again, seeking a contradiction we suppose that we have a minimal $n \in \mathbb{N}$ with $u_{1}, \ldots, u_{n} \in \bigcup \mathcal{H}$ such that $f\left(u_{1} \cdots u_{n}\right) \neq$ $u_{1} \cdots u_{n}$. Then $n>1$. By Lemma 18 we have $f\left(u_{1} \cdots u_{n}\right) \in$ $\left\{f\left(u_{1} \cdots u_{n-1}\right), f\left(u_{1} \cdots u_{n-1}\right) u_{n}\right\}$. By the minimality of $n$ we have $f\left(u_{1} \ldots u_{n-1}\right)=u_{1} \ldots u_{n-1}$, thus

$$
f\left(u_{1} \cdots u_{n}\right)=u_{1} \cdots u_{n-1} .
$$

Similarly, as $\left.f\right|_{A^{u_{n}}}=\operatorname{id}_{A^{u_{n}}}$ for all $A \in \mathcal{H}$, we have

$$
f\left(u_{1} \cdots u_{n}\right)=f\left(u_{1} \cdots u_{n-2} u_{n} u_{n-1}^{u_{n}}\right)=u_{1} \cdots u_{n-2} u_{n} .
$$

Now $u_{1} \cdots u_{n-1}=u_{1} \cdots u_{n-2} u_{n}$ implies $u_{n}=u_{n-1}$, and the proof is completed as in the previous case: $v=u_{n-1}^{2}$ is in $\bigcup \mathcal{H}$, and

$$
f\left(u_{1} \cdots u_{n}\right)=f\left(u_{1} \cdots u_{n-2} v^{2}\right)=u_{1} \cdots u_{n-2} v^{2}=u_{1} \cdots u_{n}
$$

by the minimality of $n$. The contradiction proves (4.4) and finishes the proof of the lemma.

Now we are ready to prove the most important theorem of this section.

Theorem 20. Let $G$ be a group which is generated by t-complete subgroups $A$ and $B$. Then exactly one of the following holds:

(1) $G$ is t-complete, or

(2) $A$ and $B$ are commuting, coprime torsion groups and $G=A \times B$.

Proof. Let $f$ be a compatible function on $G$ with $f(1)=1$. Then the restrictions $\left.f\right|_{A},\left.f\right|_{B}$ are compatible by Lemma 6 , and hence they are either the constant or the identity map. If $\left.f\right|_{A}=\mathrm{id}_{A}, f(B)=1$ or if $f(A)=1,\left.f\right|_{B}=\operatorname{id}_{B}$, then $A$ and $B$ are commuting, coprime 
torsion groups by Proposition $15,(3) \Rightarrow(2)$. Otherwise we have either $f(A)=1, f(B)=1$ or $\left.f\right|_{A}=\operatorname{id}_{A},\left.f\right|_{B}=\operatorname{id}_{B}$.

First let us assume that $f(A)=1, f(B)=1$. For any $g \in G$ the group $A^{g}$ is t-complete, moreover $A$ and $A^{g}$ are not coprime torsion groups. Hence $f\left(A^{g}\right)=1$ by Proposition 15 . Similarly $f\left(B^{g}\right)=1$. Thus $G$ is t-complete by Lemma 19 .

If $\left.f\right|_{A}=\mathrm{id}_{A},\left.f\right|_{B}=\mathrm{id}_{B}$, Proposition 15 yields by the same reasoning as above that $\left.f\right|_{A^{g}}=\operatorname{id}_{A^{g}}, f_{B^{g}}=\operatorname{id}_{B^{g}}$ for all $g \in G$. Again $G$ is t-complete by Lemma 19 .

Item (1) of Theorem 2 is an immediate corollary.

Corollary 21. Every group $G$ which is generated by involutions is tcomplete.

Proof. Since all four functions on $\mathbb{Z}_{2}$ are either constant or left translations, $\mathbb{Z}_{2}$ is t-complete. Let $\mathcal{H}$ be the set of subgroups of size 2 of $G$. By Proposition $15,(3) \Rightarrow(2)$, every compatible function $f$ on $G$ with $f(1)=1$ satisfies either $f(A)=1$ for all $A \in \mathcal{H}$ or $\left.f\right|_{A}=\operatorname{id}_{A}$ for all $A \in \mathcal{H}$. Since $\mathcal{H}$ is closed under conjugation by any $g \in G$, Lemma 19 yields that $G$ is t-complete.

The following groups are generated by involutions (see e.g., [11]) and consequently t-complete by Corollary 21 :

(1) for any abelian $A$, the generalized dihedral group $A \rtimes \mathbb{Z}_{2}$ with $\mathbb{Z}_{2}$ acting on $A$ by inverting elements,

(2) finite non-abelian simple groups (Odd-order Theorem [4]),

(3) and generally all Coxeter groups, i.e., groups with generators $r_{1}, \ldots, r_{k}$ satisfying the relations

$$
\left(r_{i} r_{j}\right)^{m_{i j}}=1
$$

where $m_{i i}=1$ and $m_{i j} \geq 2$ for all distinct $i, j \in\{1, \ldots, k\}$.

The Odd-order Theorem [4] together with Lemma 17 has item (2) of Theorem 2 as a consequence:

Corollary 22. Let $G$ be a finite group all of whose minimal normal subgroups are non-abelian. Then $G$ is t-complete.

Proof. Let $N$ be the product of all minimal normal subgroups of $G$. From the assumption it follows that

$N$ is a direct product of simple non-abelian groups.

Thus $N$ is t-complete by Corollary 21 .

Assume we have $g \in G \backslash N$. Let $U=\langle g\rangle$. Let $K=U N$. We claim that $K$ satisfies the assumptions of Lemma 17 with $\mathcal{H}=\left\{U^{k} \mid k \in K\right\}$. 
For $k \in K$ we have $U^{k} N=K$. Let $H=\bigcap_{k \in K} U^{k}$. Then $H$ is a cyclic normal subgroup of $K$. By (4.5) $N$ has no abelian normal subgroups and in particular $H \cap N=1$. Thus $[H, N]=1$. But the centralizer of $N$ in $G$ is trivial. Hence $H=1$ and $K$ is t-complete by Lemma 17 .

Now let $f$ be compatible on $G$ with $f(1)=1$. Since $N$ is t-complete, we have either $f(N)=1$ or $\left.f\right|_{N}=\mathrm{id}_{N}$. Since $K$ is t-complete, $f(N)=$ 1 implies $f(g)=1$ and $\left.f\right|_{N}=\operatorname{id}_{N}$ implies $f(g)=g$. As we chose $g \in G \backslash N$ arbitrarily, we obtain either $f(G)=1$ or $f=\operatorname{id}_{G}$.

Since $\mathbb{Z}_{p}$ is not t-complete for any odd prime $p$, there is no immediate generalization of Corollary 21 for groups generated by elements of order $p$. Nevertheless, we can already prove part of item (9) of Theorem 2. For that the following easy observation will be useful.

Lemma 23. Let $p$ be a prime, let $G$ be a nilpotent p-group which contains two distinct subgroups of size $p$, and let $f$ be a compatible function on $G$ with $f(1)=1$. Then there exists an integer $k$ such that for all $x \in G$ with $x^{p}=1$ we have $f(x)=x^{k}$.

Proof. Let $c \in Z(G)$ be an element of order $p$. Since $f(c) \in\langle c\rangle$, there exists an integer $k$ such that $f(c)=c^{k}$. Let $a \in G \backslash\langle c\rangle$ have order $p$. Note that such an element exists by assumption. Then $\langle c, a\rangle$ is isomorphic to $\left(\mathbb{Z}_{p}\right)^{2}$, and $f$ is a power map on $\langle c, a\rangle$ by Lemma 13. Hence $f(x)=x^{k}$ for every $x \in\langle c, a\rangle$. In particular $f$ maps every element in $\langle c\rangle$ to its $k$-th power and every element of order $p$ outside of $\langle c\rangle$ to its $k$-th power.

Corollary 24. Let $G$ be a non-abelian, nilpotent p-group that is generated by elements of prime order $p$. Then $G$ is t-complete.

Proof. We note that if $p=2$, then the statement follows from Corollary 21. Let $f$ be a compatible function on $G$ with $f(1)=1$. By Lemma 23 we have an integer $k$ such that

$$
\forall x \in G: x^{p}=1 \Rightarrow f(x)=x^{k} .
$$

In order to show $k \in\{0,1\}$ we consider a certain quotient of $G$. Let $N=[[G, G], G]$ be the third term in the lower central series $G>$ $[G, G]>[[G, G], G] \geq \ldots$ of $G$. We write $\bar{G}=G / N$ and $\bar{x}=x N$ for $x \in G$. Note that $\bar{G}^{\prime}=G^{\prime} / N$ is central in $\bar{G}$, and it is non-trivial since $G$ is non-abelian. Moreover we have $a, b \in G$ of order $p$ such that $[\bar{b}, \bar{a}] \neq 1$.

Let $f_{N}: G / N \rightarrow G / N$ be the function induced by $f$ on $G / N$ : $f_{N}(x N)=f(x) N$ for every $x \in G$. Recall that $f_{N}$ is well-defined and compatible by Lemma 7 . Let $\bar{A}=\langle\bar{a}\rangle \bar{G}^{\prime}$. Since $\bar{G}^{\prime}$ is central, $\bar{A}$ 
is an abelian normal subgroup of $\bar{G}$. In particular $\bar{A} \cap\langle\bar{b}\rangle=1$, hence $\bar{A}\langle\bar{b}\rangle$ is a semidirect product. Then Lemma 8 yields

$$
f_{N}(\bar{a} \bar{b})=f_{N}(\bar{a}) f_{N}(\bar{b})=\bar{a}^{k} \bar{b}^{k} .
$$

Further we have $r \in \mathbb{N}_{0}$ such that

$$
f_{N}(\bar{a} \bar{b})=(\bar{a} \bar{b})^{r}=[\bar{b}, \bar{a}]^{r(r-1) / 2} \bar{a}^{r} \bar{b}^{r} .
$$

For the second equality we used $\bar{G}^{\prime} \leq Z(\bar{G})$. Since $\bar{a}^{k},[\bar{b}, \bar{a}]^{r(r-1) / 2} \bar{a}^{r} \in$ $\bar{A}$, we obtain $\bar{b}^{r}=\bar{b}^{k}$. Hence $r \equiv k(\bmod p)$ and $\bar{a}^{r}=\bar{a}^{k}$ as well. Now $[\bar{b}, \bar{a}]^{r(r-1) / 2}=1$ implies that $p$ divides either $r$ or $r-1$. Thus $k \in\{0,1\}$. Finally (4.6) together with Lemma 19 yields $f(G)=1$ or $f=\operatorname{id}_{G}$.

Note that in particular the following $p$-groups are generated by elements of order $p$ and hence t-complete by Corollary 24:

(1) non-abelian nilpotent groups of exponent $p$,

(2) non-abelian semidirect products $\left(\mathbb{Z}_{p}\right)^{n} \rtimes \mathbb{Z}_{p}$.

4.3. Lifting. We investigate when the t-completeness of a group follows from the t-completeness of its quotients. First we note that a subdirect product of two t-complete groups, which is not direct, is tcomplete. We later use Theorem 25 to prove item (8) of Theorem 2.

Theorem 25. Let $G$ be a group with normal subgroups $A, B$ such that $A \cap B=1$ and $A B \neq G$. If $G / A$ and $G / B$ are $t$-complete, then $G$ is t-complete.

Proof. Let $f$ be a compatible function on $G$ with $f(1)=1$. For every normal subgroup $N$ of $G$ the induced function $f_{N}: G / N \rightarrow$ $G / N, x N \mapsto f(x) N$, is compatible on $G / N$ by Lemma 7 . By assumption, $f_{A}$ and $f_{B}$ are either constant or the identity on $G / A$ and on $G / B$, respectively. Since $A B \neq G$ the function $f$ induces either a constant or the identity on $G /(A B)$ as well.

If $f_{A B}$ is constant modulo $A B$, then both $f_{A}$ and $f_{B}$ are constant modulo $A$ and modulo $B$, respectively. Thus $f(G) \subseteq A$ and $f(G) \subseteq B$ which implies $f(G) \subseteq A \cap B=1$. Then $f$ is constant on $G$.

If $f$ induces the identity on $G /(A B)$, then $f_{A}$ is the identity on $G / A$ and $f_{B}$ is the identity on $G / B$. For $x \in G$ we have $f(x) \in$ $f(x A) \cap f(x B) \subseteq x A \cap x B=\{x\}$. Thus $f=\operatorname{id}_{G}$.

We continue by proving item (3) of Theorem 2 .

Corollary 26. A free group on at least 2 generators is t-complete. 
Proof. Let $G$ be free over some subset $X$ of $G$ with $|X|>1$. Let $f$ be a compatible function on $G$ with $f(1)=1$. We will prove that

either $f\left(\langle a\rangle^{g}\right)=1 \forall a \in X \forall g \in G$ or $\left.f\right|_{\langle a\rangle^{g}}=\operatorname{id}_{\langle a\rangle^{g}} \forall a \in X \forall g \in G$.

Let $N$ be the normal subgroup of $G$ that is generated by $\left\{a^{2} \mid a \in X\right\}$. Since $G / N$ is generated by involutions, it is t-complete by Corollary 21 . Then the induced function $f_{N}: G / N \rightarrow G / N, x N \mapsto f(x) N$, is compatible on $G / N$ by Lemma 7 . So $f_{N}$ is either constant or the identity on $G / N$. Assume that $f(G) \subseteq N$. Let $a, b \in X$ with $a \neq b$, and let $H=\langle a, b\rangle$. Then $f(a b) \in\langle a b\rangle \cap N=1$ yields $f(a b)=1$. We note that $f$ induces a power map on $H / H^{\prime} \cong \mathbb{Z}^{2}$ by Lemma 13 . So $f(a b)=1$ implies $f(a) \in\langle a\rangle \cap H^{\prime}=1$. Thus $f(a)=1$. Similarly, we obtain $f\left(\langle a\rangle^{g}\right)=1$ for all $g \in G$. If $f_{N}$ is the identity on $G / N$, then $\left.f\right|_{\langle a\rangle^{g}}=\operatorname{id}_{\langle a\rangle^{g}}$ for all $g \in G$ follows in the same fashion. This proves (4.7). Now Lemma 19 yields that $G$ is t-complete.

The following lemma is used for proving items (4) and (5) of Theorem 2 .

Lemma 27. Let $G$ be a group, let $N$ be a normal subgroup of $G$, and let $\mathcal{H}=\{H \leq G \mid N \cap H=1\}$. Assume that $\langle\bigcup \mathcal{H}\rangle=G$ and that $G / N$ is t-complete. Then $G$ is t-complete

Proof. Let $f$ be a compatible function on $G$ with $f(1)=1$. Then the induced function $f_{N}: G / N \rightarrow G / N, x N \mapsto f(x) N$, is compatible on $G / N$ by Lemma 7 . By assumption, $f_{N}$ is either constant or the identity on $G / N$.

If $f_{N}$ is constant, then $f(G) \subseteq N$ and

$$
f(H) \subseteq H \cap N=1 \text { for all } H \in \mathcal{H} .
$$

If $f_{N}$ is the identity on $G / N$, then for all $H \in \mathcal{H}, h \in H$ we have $f(h) \subseteq H \cap h N=\{h\}$. Hence

$$
\left.f\right|_{H}=\mathrm{id}_{H} \text { for all } H \in \mathcal{H} .
$$

Since $\mathcal{H}$ is closed under conjugation by any $g \in G$, Lemma 19 yields that $G$ is t-complete.

Recall that a group is perfect if it is equal to its derived subgroup. Item (4) of Theorem 2 is now a consequence of the previous Lemma 27 and of Corollary 22:

Corollary 28. Let $G$ be a finite perfect group. Then $G$ is t-complete. 
Proof. We prove the statement by induction on the size of $G$. If all the minimal normal subgroups of $G$ are non-abelian, then $G$ is t-complete by Corollary 22.

Now let us assume that $N$ is an abelian minimal normal subgroup of $G$. Then $N$ is an elementary abelian $p$-group for some prime $p$. Let $\mathcal{M}$ be the set of cyclic subgroups of $G$ whose orders are coprime to $p$. Then $M=\langle\bigcup \mathcal{M}\rangle$ is normal in $G$, and $G / M$ is a finite $p$-group. Since every quotient of a perfect group is perfect, this implies $G=M$. Hence $G$ is generated by subgroups which intersect with $N$ trivially. Since $G / N$ is t-complete by the induction assumption, Lemma 27 yields that $G$ is t-complete as well.

As further consequence of Lemma 27 we obtain item (5) of Theorem 2:

Corollary 29. For $n>1$ and for a field $F$, every group $G$ with $\mathrm{SL}(n, F) \leq G \leq \mathrm{GL}(n, F)$ is t-complete.

Proof. We note that $\mathrm{SL}\left(2, \mathbb{Z}_{2}\right)=\mathrm{GL}\left(2, \mathbb{Z}_{2}\right) \cong S_{3}$ is generated by involutions and hence t-complete by Corollary 21 . In the following we assume that $n>2$ or $|F|>2$. Let $S=\operatorname{SL}(n, F)$, and let $Z=Z(S)$. We claim that

$$
S / Z \text { is t-complete. }
$$

Note that $\operatorname{SL}\left(2, \mathbb{Z}_{3}\right) / Z \cong A_{4}$ is t-complete by Lemma 17 : the Klein 4 -group $N \unlhd A_{4}$ is an elementary abelian 2-group and hence t-complete. Moreover the Sylow 3-subgroups of $A_{4}$ are complements to $N$ and their intersection is trivial. Assume that $n>2$ or $|F|>3$. We show that $S / Z$ is generated by involutions. We denote the block diagonal matrix with square matrices $A_{1}, \ldots, A_{k}$ along the diagonal and 0 elsewhere by $\operatorname{diag}\left(A_{1}, \ldots, A_{k}\right)$. Define an $n \times n$-matrix

$$
s= \begin{cases}\operatorname{diag}\left(\left(\begin{array}{cc}
0 & -1 \\
1 & 0
\end{array}\right), 1, \ldots, 1\right) & \text { if } n=2 \text { or } F \text { has characteristic } 2, \\
\operatorname{diag}(-1,-1,1, \ldots, 1) & \text { otherwise. }\end{cases}
$$

Here $s \in S \backslash Z$ and $s^{2} \in Z$. Then the conjugates of $s Z$ are involutions, which generate a normal subgroup of $S / Z$. Since $S / Z$ is simple [11, 3.2.9], this implies that $S / Z$ is generated by involutions. So (4.8) follows from Corollary 21.

Next we show that

$S$ is t-complete.

For $i, j \in\{1, \ldots, n\}$ with $i \neq j$, let $e_{i j}$ denote the matrix with 1 in the $(i, j)$-th position and 0 everywhere else. For $a \in F \backslash\{0\}$ a matrix of the form $t=1+a e_{i j}$ is called a transvection. Note that every power of 
a transvection is either a transvection or 1 . In particular $\langle t\rangle \cap Z=1$ for every transvection $t \in S$. Since $S$ is generated by its transvections by [11, 3.2.10], Lemma 27 implies that $S$ is t-complete.

Finally we note that for every subgroup $G$ of $\mathrm{GL}(n, F)$ that contains $S$, there exists a subgroup $H$ of the multiplicative group of $F$ (the determinants of the elements of $G$ ) such that

$$
G=S\{\operatorname{diag}(h, 1, \ldots, 1) \mid h \in H\}=S\{\operatorname{diag}(1, \ldots, 1, h) \mid h \in H\} .
$$

Since $S$ is t-complete by (4.9) and has two complements in $G$ which intersect trivially, Lemma 17 yields that $G$ is t-complete.

4.4. Partitioned groups. A group $G$ is partitioned by a set $\mathcal{H}$ of proper subgroups of $G$ if every $x \in G \backslash\{1\}$ is contained in exactly one $H \in \mathcal{H}$. For an overview and the complete classification of finite partitioned groups we refer to [15].

Recall that a Frobenius group is a transitive permutation group such that no non-trivial element fixes more than one point and there exist an element fixing exactly one point. The stabilizer of a point is called a Frobenius complement, the elements not fixing any point together with the identity element form the Frobenius kernel. The Frobenius kernel is a normal subgroup in the Frobenius group (see, e.g., [11]). Every Frobenius group is partitioned by the kernel and the conjugates of the complement. Other examples for partitioned groups are $p$-groups of exponent $p$. Non-abelian nilpotent $p$-groups of exponent $p$ are tcomplete by Corollary 24. In this section we investigate some properties of compatible functions on finite non-abelian partitioned groups. We continue our investigation with semidirect products in Section 4.5, then investigate groups generated by elements of prime order in Section 4.6. In Section 4.7 we revisit partitioned groups and prove that all finite non-abelian partitioned groups are t-complete. The following lemma is crucial for the proof.

Lemma 30. Let $G$ be a group that is partitioned by a set $\mathcal{H}$ of proper subgroups, and let $f$ be a compatible function on $G$ such that $f(1)=1$.

(1) If there exists an $a \in G \backslash\{1\}$ with $f(a)=1$, then $f(G)=1$.

(2) If there exists an $a \in G \backslash\{1\}$ with $f(a)=a$, then $f=\operatorname{id}_{G}$.

Proof. Let $a \in A$ with $A \in \mathcal{H}$ such that $f(a)=1$ or $f(a)=a$. Let $b \in B$ for some $B \in \mathcal{H}, B \neq A$. Let $C$ be the unique element in $\mathcal{H}$ such that $a^{-1} b \in C$. Note that $C$ is neither $A$ nor $B$. The intersection of any two cosets corresponding to distinct subgroups in $\mathcal{H}$ has at most one element since any two such subgroups meet in 1 . Therefore $f(b)$ is uniquely determined by its $B$-coset $B$ and its $C$-coset $f(b) C$. Since 
$a C=b C$, we have $f(a) C=f(b) C$. Thus

$$
f(a) C \cap B=\{f(b)\} .
$$

Case 1: If $f(a)=1$, then $f(a) C \cap B=\{1\}$ and $f(b)=1$. Hence $f(G \backslash A)=1$. When switching the role of $a$ and $b$ the same argument yields $f(G \backslash B)=1$. Thus $f(G)=1$.

Case 2: If $f(a)=a$, then $f(a) C \cap B=\{b\}$. Hence $f(b)=b$ and $\left.f\right|_{G \backslash A}=\operatorname{id}_{G \backslash A}$. When switching the role of $a$ and $b$ the same argument yields $\left.f\right|_{G \backslash B}=\operatorname{id}_{G \backslash B}$. Thus $f=\operatorname{id}_{G}$.

Lemma 30 immediately yields that every partitioned group which contains some t-complete subgroup (e.g., a subgroup of size 2) is tcomplete. Another consequence is the following.

Corollary 31. Let $G$ be a partitioned group, and let $f$ be a compatible function on $G$. Then $f$ is either constant or injective.

Proof. Assume we have distinct elements $a, b$ in $G$ such that $f(a)=$ $f(b)$. Then $h: G \rightarrow G, x \mapsto f(a)^{-1} f(a x)$, is compatible and satisfies $h(1)=1=h\left(a^{-1} b\right)$. Hence $h(G)=1$ by Lemma 30. Now $f(G)=f(a)$ follows.

4.5. Semidirect products. Next we show that the smallest nonabelian partitioned groups are t-complete:

Lemma 32. Let $G$ be a non-abelian group of size pq for distinct primes $p$ and $q$. Then $G$ is t-complete.

Proof. Even though $G$ is not commutative, we use + to denote the group operation. We denote the identity element of $G$ by 0 . Without loss of generality we may assume that $G$ is generated by elements $a, b$ with $\operatorname{ord}(a)=p, \operatorname{ord}(b)=q$, and $b+a-b=r a$ for some $r \in\{2, \ldots, p-$ $1\}$. Note that $r^{q} \equiv 1(\bmod p)$. Hence $G$ is the semidirect product of the normal subgroup $\langle a\rangle$ of order $p$ and a complement $\langle b\rangle$ of order $q$ with $q<p$. In particular $G$ is partitioned by $\langle a\rangle$ and all the conjugates of $\langle b\rangle$.

Let $f$ be a compatible function with $f(0)=0$ and $f(G) \neq 0$. We prove that $f=\mathrm{id}_{G}$. By Lemma 30 it suffices to show that $f$ has a fixed point in $G \backslash\{0\}$. To this end, let $k \in\{1, \ldots, q-1\}$ and consider first $f(k b)$. Since $f(k b)$ is contained in the subgroup $\langle b\rangle$ of size $q$ and $f$ is not constant, by Corollary 31 there is a bijection $\alpha:\{1, \ldots, q-1\} \rightarrow$ $\{1, \ldots, q-1\}$ such that

$$
f(k b)=\alpha(k) b
$$


Now consider $f(a+k b)$. Then $f(a+k b)$ is contained in the subgroup $\langle a+k b\rangle$ of size $q$. If $f(a+k b)=0$, then $f(G)=0$ by Lemma 30. Hence we have some $n \in\{1, \ldots, q-1\}$ (depending on $k$ ) such that

$$
\begin{aligned}
f(a+k b) & =n(a+k b) \\
& =a+(k b+a-k b)+\ldots+(k(n-1) b+a-k(n-1) b)+k n b \\
& =a+r^{k} a+\ldots+r^{k(n-1)} a+k n b,
\end{aligned}
$$

thus

$$
f(a+k b)=\frac{r^{k n}-1}{r^{k}-1} a+k n b .
$$

By Lemma 8 we have $f(a+k b)=f(a)+f(k b)$. This is the unique way to split $f(a+k b)$ into a sum of an element of $\langle a\rangle$ and an element of $\langle b\rangle$. Together with equations (4.10) and (4.11) we obtain

$$
f(k b)=k n b=\alpha(k) b,
$$

and thus

$$
f(a)=\frac{r^{\alpha(k)}-1}{r^{k}-1} a .
$$

If $f(a)=0$, then $f(G)=0$ by Lemma 30. Hence by (4.12), we have $m \in$ $\{1, \ldots, p-1\}$ such that $\frac{r^{\alpha(k)}-1}{r^{k}-1} \equiv m(\bmod p)$ for all $k \in\{1, \ldots, q-1\}$. Thus

$$
r^{\alpha(k)}-1 \equiv m\left(r^{k}-1\right) \quad(\bmod p) \text { for all } k \in\{1, \ldots, q-1\} .
$$

Taking the sum of all these equations yields

$$
\sum_{k=1}^{q-1}\left(r^{\alpha(k)}-1\right) \equiv m \sum_{k=1}^{q-1}\left(r^{k}-1\right) \quad(\bmod p) .
$$

Since $\alpha:\{1, \ldots, q-1\} \rightarrow\{1, \ldots, q-1\}$ is a bijection, (4.13) yields

$$
(m-1) \sum_{k=0}^{q-1}\left(r^{k}-1\right) \equiv 0 \quad(\bmod p) .
$$

Together with $\sum_{k=0}^{q-1} r^{k} \equiv \frac{r^{q}-1}{r-1} \equiv 0(\bmod p)$ we obtain

$$
(m-1)(-q) \equiv 0 \quad(\bmod p)
$$

Since $q<p$, this means $m \equiv 1(\bmod p)$. From $(4.12)$ we derive $f(a)=m a=a$. Thus $f$ has a non-trivial fixed point and $f=\operatorname{id}_{G}$ by Lemma 30 .

Corollary 33. Let $p, q$ be primes, let $n \in \mathbb{N}$. Let $G=\left(\mathbb{Z}_{p}\right)^{n} \rtimes \mathbb{Z}_{q}$ be non-abelian. Then $G$ is t-complete. 
Proof. If $p=q$, then $G$ is t-complete by Corollary 24. If $n=1$, then $G$ is t-complete by Lemma 32. We assume $p \neq q$ and $n \geq 2$ in the following. First we construct a subgroup $H$ of $G$ that is partitioned. Let $N$ be the normal Sylow $p$-subgroup of $G$, let $B$ be a Sylow $q$ subgroup. By the conjugation action of $B$ on $N$, we may consider $N$ as module over the group algebra $\mathbb{Z}_{p}[B]$.

As $p \neq q$, Maschke's Theorem [11, 8.1.2] yields that $N$ is a direct sum of simple $\mathbb{Z}_{p}[B]$-modules. Since $G$ is non-abelian, $N$ is a faithful $\mathbb{Z}_{p}[B]$-module. Hence $N$ contains a non-trivial simple $\mathbb{Z}_{p}[B]$-module $M$. Since every $b \in B \backslash\{1\}$ generates $B$, we have $C_{M}(b)=C_{M}(B)=1$. Consequently $B^{m} \cap B=1$ for all $m \in M \backslash\{1\}$. Let $H=M B$, then $H$ is a Frobenius group with complement $B$ and kernel $M$. In particular $H$ is partitioned; $M$ and $B^{m}$ for $m \in M$ form a partition of $H$.

Let $f$ be a compatible function $f$ on $G$ with $f(1)=1$. We claim that

$$
f(H)=1 \text { or }\left.f\right|_{H}=\operatorname{id}_{H} \text {. }
$$

Assuming $f(H) \neq 1$, we want to show that $f$ has a fixed point in $H \backslash\{1\}$. Let $a \in M \backslash\{1\}, b \in B \backslash\{1\}$. Now $a b\left\langle a^{b}\right\rangle=b\left\langle a^{b}\right\rangle$ implies $f(a b)\left\langle a^{b}\right\rangle=f(b)\left\langle a^{b}\right\rangle$. Hence

$$
f(b)^{-1} f(a b) \in\langle a\rangle^{b} .
$$

Since $f(a b)=f(a) f(b)$ by Lemma 8 , this yields $f(a)^{f(b)} \in\langle a\rangle^{b}$ and finally

$$
f(a)^{f(b) b^{-1}} \in\langle a\rangle .
$$

If $f(a)=1$, then $f(H)=1$ by Lemma 30. Thus $f(a)$ generates $\langle a\rangle$. Consequently (4.15) implies that $f(b) b^{-1}$ normalizes $\langle a\rangle$.

If $f$ fixes $b$, then $f=\operatorname{id}_{H}$ by Lemma 30. Assume $f(b) b^{-1} \neq 1$. Since $f(b) b^{-1}$ generates $B$, we then have that $b$ normalizes $\langle a\rangle$. Hence $\langle a, b\rangle=H$ is a non-abelian subgroup of size $p q$. Lemma 32 yields $\left.f\right|_{H}=\mathrm{id}_{H}$, and (4.14) is proved.

Since $\left.f\right|_{N}$ is a power map by Lemma 13, (4.14) implies $f(N)=$ $1, f(B)=1$ or $\left.f\right|_{N}=\mathrm{id}_{N},\left.f\right|_{B}=\mathrm{id}_{B}$. Now the result follows from Lemma 8.

Item (6) of Theorem 2 is an easy consequence of Corollary 33:

Corollary 34. Let $G$ be a semidirect product of a normal elementary abelian subgroup $A$ and a complement $B$ such that $C_{B}(A)=1$. Then $G$ is t-complete.

Proof. Let $b \in B$ have prime order. Then $A\langle b\rangle$ is non-abelian and hence t-complete by Corollary 33. Every compatible function $f$ on $G$ with $f(1)=1$ restricts to a constant or the identity on $A\langle b\rangle$. Since 
the intersection of the complements of $A$, namely $\bigcap_{a \in A} B^{a}$, is a normal subgroup of $G$ that intersects $A$ trivially, we have $\bigcap_{a \in A} B^{a} \subseteq C_{B}(A)=$ 1. The result now follows from Lemma 17.

Let us recall that a transitive permutation group $G$ on a finite set $\Omega$ is primitive if $G$ preserves no non-trivial partition of $\Omega$. Equivalently, a transitive group $G$ is primitive if and only if the stabilizer $G_{\alpha}$ of some element $\alpha \in \Omega$ is a maximal subgroup of $G$. Every 2-transitive group is primitive.

We are now ready to prove (7) of Theorem 2 .

Corollary 35. Let $G$ be a finite non-abelian primitive permutation group on $\Omega$. Then $G$ is t-complete.

Proof. If all minimal normal subgroups of $G$ are non-abelian, then the result follows from Corollary 22. Hence assume that $G$ has an abelian minimal normal subgroup $N$. Let $\alpha \in \Omega$. By $[11,7.2 .6] G$ is a semidirect product of $N$ and the point stabilizer $G_{\alpha}$. Further $C_{G_{\alpha}}(N)=1$. Now Corollary 34 yields the result.

Recall that every finite group $G$ has quotients with primitive actions: Let $M$ be a maximal subgroup of $G$, and let $M_{G}=\bigcap_{g \in G} M^{g}$ be the core of $M$ in $G$. Then $G / M_{G}$ acts by left multiplication on the left cosets of $M$ in $G$. This action is faithful, and it is primitive because the point stabilizer $M / M_{G}$ of $M$ is a maximal subgroup in $G / M_{G}$. By Corollary 35 we have that $G / M_{G}$ is t-complete except if it is abelian, that is, if $M$ contains the derived subgroup $G^{\prime}$ and thus $M=M_{G}$ has prime index in $G$.

We can now prove (8) of Theorem 2.

Theorem 36. Let $G$ be a finite group. Then $G / \operatorname{Frat}(G)$ splits into a direct product of pairwise coprime subgroups that are elementary abelian or t-complete.

Proof. We use induction on the size of $G / \operatorname{Frat}(G)$. Let $N$ be a normal subgroup of $G$ that is minimal such that $\operatorname{Frat}(G) \leq N$ and $G / N$ is t-complete. If $N=G$, then $G / M_{G}$ is simple and abelian for every maximal subgroup $M$ of $G$ by Corollary 35. Hence every maximal subgroup $M$ of $G$ contains $G^{\prime}$, and $G / \operatorname{Frat}(G)$ is a direct product of elementary abelian subgroups.

We assume $N<G$ in the following. Let $M$ be a maximal subgroup of $G$. Assume $\operatorname{gcd}\left(|G: N|,\left|G: M_{G}\right|\right)>1$. We claim

$$
N \subseteq M \text {. }
$$

Case 1. If $G / M_{G}$ is abelian, then $M_{G}=M$ is a maximal subgroup in $G$ and either $N \subseteq M$ or $N M_{G}=G$. In the latter case $G /(N \cap$ 
$\left.M_{G}\right) \cong G / N \times G / M_{G}$ is t-complete by Proposition 16 . This implies $N \subseteq M_{G} \subseteq M$ by the minimality of $N$.

Case 2. If $G / M_{G}$ is t-complete, then either $N M_{G}<G$ and $G /(N \cap$ $\left.M_{G}\right)$ is t-complete by Theorem 25 or $N M_{G}=G$ and $G /\left(N \cap M_{G}\right) \cong$ $G / N \times G / M_{G}$ is t-complete by Theorem 20. Thus $G /\left(N \cap M_{G}\right)$ is t-complete which yields that $N \subseteq M_{G} \subseteq M$ by the minimality of $N$. Hence (4.16) is proved.

Let $S$ be the set of maximal subgroups of $G$ that do not contain $N$, and let

By (4.16) we have

$$
K=\bigcap S
$$

$$
\operatorname{Frat}(G)=N \cap K \text { and } \operatorname{gcd}(|G: N|,|G: K|)=1 .
$$

By the induction hypothesis $G / K$ splits into a direct product of pairwise coprime subgroups $M_{1}, \ldots, M_{n}$ that are elementary abelian or t-complete. Hence

$$
G / \operatorname{Frat}(G) \cong M_{1} \times \ldots \times M_{n} \times G / N
$$

with all factors pairwise coprime and elementary abelian or t-complete.

4.6. Groups generated by elements of prime order. Corollary 33 helps us to omit the assumption of nilpotence in Corollary 24.

Lemma 37. Let $p$ be a prime, and let $G$ be a finite non-abelian group that is generated by elements of order $p$. Then $G$ is t-complete.

Proof. If $p=2$, then the lemma follows from Corollary 21. We assume that $p>2$ in the following. If $G$ is perfect, then the result follows from Corollary 28. Assume that $G / G^{\prime}$ is a non-trivial elementary abelian $p$-group. Let $f$ be a compatible function on $G$ such that $f(1)=1$. First we prove that

(4.17) $\exists A \leq G,|A|=p, A \cap G^{\prime}=1$ : either $f(A)=1$ or $\left.f\right|_{A}=\operatorname{id}_{A}$.

Case 1, $G^{\prime}$ is perfect: Then $G^{\prime}$ is t-complete by Corollary 28. Let $P$ be a Sylow $p$-subgroup of $G$. First assume $P \cap G^{\prime} \neq 1$. Then we have $H \leq P \cap G^{\prime}$ with $|H|=p$ such that either $f(H)=1$ or $\left.f\right|_{H}=\operatorname{id}_{H}$. Since $P$ also contains a subgroup $A$ of size $p$ that is not contained in $G^{\prime}$, Lemma 23 yields (4.17). Next we assume $P \cap G^{\prime}=1$. Since $P$ cannot be normal in $G$, we have an $A \leq P$ with $|A|=p$ and $g \in G^{\prime}$ such that $A^{g} \nsubseteq P$. Then $A \cap A^{g}=1$ and $G^{\prime} A$ is t-complete by Lemma 17 . This implies (4.17).

Case 2, $G^{\prime \prime}<G^{\prime}, G / G^{\prime \prime}$ is nilpotent: Now $G / G^{\prime \prime}$ is a non-abelian $p$-group generated by elements of order $p$. Hence $G / G^{\prime \prime}$ is t-complete 
by Corollary 24. Let $A \leq G$ be any subgroup of order $p$ such that $A \cap G^{\prime}=1$. Now (4.17) follows from Lemma 7 .

Case 3, $G^{\prime \prime}<G^{\prime}, G / G^{\prime \prime}$ is not nilpotent: Since $G / G^{\prime \prime}$ is solvable, we then have normal subgroups $B, C$ of $G$ (two neighbouring elements of a principal series) with $G^{\prime \prime} \leq B<C \leq G^{\prime}$ such that $C / B$ is a minimal normal subgroup of $G / B$ but is not central in $G / B$. Then $C / B \cong\left(\mathbb{Z}_{q}\right)^{n}$ for some prime $q$ and some $n \in \mathbb{N}$. Further we have some $a \in G \backslash G^{\prime}$ with $\operatorname{ord}(a)=p$ that does not centralize $C / B$. Now $U=$ $C\langle a\rangle / B$ is isomorphic to a non-abelian semidirect product $\left(\mathbb{Z}_{q}\right)^{n} \rtimes \mathbb{Z}_{p}$. By Corollary 33, $f$ induces either the constant function on $U$ or the identity. In particular (4.17) follows for $A=\langle a\rangle$. The proof of (4.17) is complete.

First consider the case that we have $A \leq G$ with $|A|=p$ and $A \cap G^{\prime}=$ 1 such that $\left.f\right|_{A}=\operatorname{id}_{A}$. For $g \in G, a \in A$ we have $a^{g} G^{\prime}=a G^{\prime}$, which yields $f\left(a^{g}\right) \in A^{g} \cap f(a) G^{\prime}=\left\{a^{g}\right\}$. Hence $f$ acts as identity mapping on every conjugate of $A$ in $G$. On each Sylow $p$-subgroup $P$ of $G$, Lemma 23 yields that for all $x \in P$ with $x^{p}=1$ we have $f(x)=x$. Hence $f$ maps every element of order $p$ in $G$ to itself. Thus $f=\operatorname{id}_{G}$ by Lemma 19.

Now let $A \leq G$ with $|A|=p$ and $A \cap G^{\prime}=1$ such that $f(A)=1$. For $g \in G$ we have $f\left(A^{g} G^{\prime}\right)=f\left(A G^{\prime}\right) \subseteq G^{\prime}$, which yields $f\left(A^{g}\right) \subseteq$ $A^{g} \cap G^{\prime}=1$. Since every Sylow $p$-subgroup $P$ of $G$ contains some conjugate of $A$ in $G$, Lemma 23 yields that for all $x \in P$ with $x^{p}=1$ we have $f(x)=1$. Hence $f$ maps every element of order $p$ in $G$ to 1 . Thus $f(G)=1$ by Lemma 19 .

Lemma 37 together with Corollary 24 yields (9) of Theorem 2:

Theorem 38. Let $G$ be a finite group that is generated by its elements of prime order. Then $G$ splits into a direct product of pairwise coprime subgroups that are elementary abelian or t-complete.

Proof. We use induction on the size of $G$. For a prime $p$ let

$$
\Omega_{p}(G)=\left\langle x \in G \mid x^{p}=1\right\rangle .
$$

By assumption

$$
G=\prod\left\{\Omega_{p}(G) \mid p \text { prime }\right\}
$$

If $p=2$, then $\Omega_{p}(G)$ is t-complete by Corollary 21. If $p$ is odd, then $\Omega_{p}(G)$ is either elementary abelian or non-abelian, t-complete by Lemma 37.

Let $N$ be a normal subgroup of $G$ that is maximal such that $N$ is tcomplete. If $N=1$, then $\Omega_{p}(G)$ is elementary abelian for every prime 
p. Moreover $G$ splits into a direct product of the subgroups $\Omega_{p}(G)$ by (4.18).

We assume $N>1$ in the following. First we show that

$$
\Omega_{p}(G) \cap N \in\left\{1, \Omega_{p}(G)\right\} \text { for every prime } p .
$$

Let $p$ be a prime. Assume that $\Omega_{p}(G) \cap N \neq 1$. If $\Omega_{p}(G)$ is t-complete, then $\Omega_{p}(G) N$ is t-complete by Theorem 20. Otherwise, if $\Omega_{p}(G)$ is elementary abelian, then every compatible function $f$ on $\Omega_{p}(G) N$ with $f(1)=1$ is constant or the identity on $\Omega_{p}(G)$ by $\Omega_{p}(G) \cap N \neq 1$ and Lemma 13. Further $\Omega_{p}(G) N$ is t-complete by Lemma 19. In any case the maximality of $N$ yields $\Omega_{p}(G) \subseteq N$. Hence (4.19) is proved.

Let $S$ be the set of primes $p$ such that $\Omega_{p}(G) \cap N=1$, and let

$$
M=\prod_{p \in S} \Omega_{p}(G)
$$

By (4.19) we have

$$
G=M N \text { and } \operatorname{gcd}(|M|,|N|)=1 .
$$

Since $M$ is normal in $G$, we obtain $G=M \times N$. By the induction hypothesis $M$ splits into a direct product of pairwise coprime subgroups $M_{1}, \ldots, M_{n}$ that are elementary abelian or t-complete. Hence

$$
G \cong M_{1} \times \ldots \times M_{n} \times N
$$

with all factors pairwise coprime and elementary abelian or t-complete.

The result above immediately implies the following.

Corollary 39. Every finite group that is generated by two noncommuting elements of prime order is t-complete.

4.7. Partitioned groups revisited. Finally we are ready to prove (10) of Theorem 2 as a consequence of Lemma 37.

Corollary 40. Let $G$ be a finite non-abelian group with a partition $\mathcal{H}$. Then $G$ is t-complete.

Proof. If $G$ contains an involution, the result follows from Lemma 30 . We assume that $|G|$ is odd in the following. By the Odd-order theorem $G$ is solvable [4]. We have an abelian minimal normal subgroup $A$ of $G$, which is isomorphic to $\left(\mathbb{Z}_{p}\right)^{n}$ for some odd prime $p$ and $n \in \mathbb{N}$. Let $H_{p}(G)=\left\langle x \in G \mid x^{p} \neq 1\right\rangle$ be the Hughes subgroup. The idea of the proof is that if $G$ is generated by elements of prime order then we use Lemma 37; otherwise we find a subgroup isomorphic to $\left(\mathbb{Z}_{p}\right)^{n} \rtimes \mathbb{Z}_{q}$ and use Corollary 33 and Lemma 30. 
Case 1: Assume that $|G|$ has a prime divisor $q$ distinct from $p$, and that there exists an element $b \in G$ whose order is a power of $q$ such that $b$ does not centralize $A$ but $b^{q}$ does. Then $U=A\langle b\rangle /\left\langle b^{q}\right\rangle$ is nonabelian and isomorphic to $\left(\mathbb{Z}_{p}\right)^{n} \rtimes \mathbb{Z}_{q}$. Let $f$ be a compatible function on $G$ with $f(1)=1$. By Corollary 33 the function $f$ induces either the constant function on $U$ or the identity. In particular $f(A)=1$ or $\left.f\right|_{A}=\operatorname{id}_{A}$ which implies that $G$ is t-complete by Lemma 30 .

Case 2: Assume that $|G|$ has a prime divisor $q$ distinct from $p$, and that all elements in $G$ whose orders are not divisible by $p$ centralize $A$. We prove that $H_{p}(G) \leq H$ for some $H \in \mathcal{H}$. Let $a \in A \backslash\{1\}$, and let $g \in G$ with $\operatorname{ord}(g)$ coprime to $p$. Since $(g a)^{p}=g^{p} \neq 1$, we have that $g, g a$, and thus $a$ are in the same subgroup $H \in \mathcal{H}$. Consequently $A \subseteq H$ and all elements with orders not divisible by $p$ are contained in $H$. Now let $g \in G$ with $\operatorname{ord}(g)=p^{m}$ for some $m>1$. Thus $C=\langle g\rangle$ is a $p$-group that acts by conjugation on $A$. Hence $A$ is partitioned into $C$-orbits which all have $p$-power size. Since $A$ is a $p$-group itself, not all orbits distinct from 1 have size divisible by $p$. Hence there exists another 1-element orbit, say the orbit of the non-trivial element $a \in A$. Then $a$ commutes with $g$. Again $(g a)^{p}=g^{p} \neq 1$ yields that $g a, g$, and $a$ are contained in the same component of $\mathcal{H}$, namely $H$. Hence $H_{p}(G) \subseteq H$ and in particular $H_{p}(G) \neq G$. Since $G$ is covered by $H_{p}(G)$ and $\left\langle G \backslash H_{p}(G)\right\rangle$, these two subgroups cannot be proper at the same time. Thus $G$ is generated by the elements of $G \backslash H_{p}(G)$, which have order $p$. Since $G$ is not abelian, Lemma 37 yields that $G$ is t-complete.

Case 3: Assume that $G$ is a $p$-group. We prove that $H_{p}(G) \leq H$ for some $H \in \mathcal{H}$. Since $G$ is a $p$-group, $A$ is central. Let $a \in A \backslash\{1\}$, and let $g \in G$ with $\operatorname{ord}(g)>p$. Since $(g a)^{p}=g^{p} \neq 1$, we have that $g, g a$, and thus $a$ are in the same subgroup $H \in \mathcal{H}$. Hence all elements of order greater than $p$ are contained in $H$. Thus $H_{p}(G) \subseteq H$ and in particular $H_{p}(G) \neq G$. Since $G$ is covered by $H_{p}(G)$ and $\left\langle G \backslash H_{p}(G)\right\rangle$, these two subgroups cannot be proper at the same time. Thus $G=\left\langle G \backslash H_{p}(G)\right\rangle$, and therefore $G$ is generated by elements of order $p$. Since $G$ is not abelian by assumption, Lemma 37 yields that $G$ is t-complete.

The proof of Theorem 2 is complete.

4.8. Maximal t-complete quotients. For the proof of Theorem 36 we used that for every finite group $G$ with maximal subgroup $M$ the action of $G / M_{G}$ on the left cosets of $M$ is primitive. Note that $G / M_{G}$ is abelian if and only if $G^{\prime} \subseteq M$. Otherwise $G / M_{G}$ is t-complete by Corollary 35. All maximal subgroups of $G$ contain $G^{\prime}$ (i.e., the Frattini subgroup of $G$ contains $G^{\prime}$ ) if and only if $G$ is nilpotent [11, 5.2.16]. 
Thus every finite group that is not nilpotent has some non-trivial tcomplete quotient.

By Theorem 3 we find the following examples of groups that do not have any non-trivial t-complete quotients at all: abelian groups of odd order and powerful $p$-groups for any odd prime $p$.

In this section we want to investigate t-complete quotients of groups in general. To this end we define

$$
\mathcal{N}(G)=\{N \unlhd G \mid G / N \text { is t-complete }\}
$$

and

$$
T(G)=\bigcap \mathcal{N}(G)
$$

Theorem 41. For every group $G$ we have the following:

(1) Every element in $\mathcal{N}(G)$ contains a minimal element in $\mathcal{N}(G)$; further every minimal element in $\mathcal{N}(G)$ is characteristic in $G$.

(2) $T(G)$ is characteristic in $G$.

(3) If $G$ is t-complete, then $T(G)=1$.

(4) For $G$ finite, we have $T(G)=G$ if and only if $G$ is a direct product of powerful p-groups for odd primes $p$.

(5) $G / T(G)$ is either t-complete or a subdirect product of $t$ complete, pairwise coprime torsion groups.

Proof. For (1) let $\mathcal{C}$ be a subset of $\mathcal{N}(G)$ that is linearly ordered by inclusion. We claim that

$$
G / \bigcap \mathcal{C} \text { is t-complete. }
$$

Let $f$ be compatible on $G$ with $f(1)=1$. Then $f$ induces a compatible function $f_{N}$ on $G / N$ for all $N \in \mathcal{C}$. Hence either $f(G) \subseteq N$ for all $N \in \mathcal{C}$ or $f(x) \in x N$ for all $x \in G, N \in \mathcal{C}$. Consequently $f(G) \subseteq \bigcap \mathcal{C}$ or $f(x) \in \bigcap_{N \in \mathcal{C}} x N=x \bigcap \mathcal{C}$ for all $x \in G$. Thus we have (4.20), and the first part of (1) follows.

Next let $N$ be a minimal element in $\mathcal{N}(G)$, and let $\alpha$ be an automorphism of $G$. Then $G / \alpha(N)$ is isomorphic to $G / N$ and consequently t-complete. Let $M=N \cap \alpha(N)$. If $\alpha(N) N=G$, then $G / M$ is isomorphic to $(G / N)^{2}$. Hence $G / M$ is t-complete by Theorem 20. If $\alpha(N) N<G$, then $G / M$ is t-complete by Theorem 25 . Thus $M=N$ by the minimality of $N$. Hence $N$ is characteristic.

Items (2) and (3) are immediate from the definition of $T(G)$.

For proving (4) we first assume that $T(G)=G$ for a finite group $G$. Then $G / \operatorname{Frat}(G)$ is abelian by Theorem 36 , and $G$ is nilpotent by $[11,5.2 .16]$. Further $G$ cannot have a quotient of size 2 . Hence $|G|$ is odd. For every prime divisor $p$ of $|G|$ the quotient $G /\left\langle x^{p} \mid x \in G\right\rangle$ has exponent $p$ and is t-complete or abelian by Theorem 38. Thus 
$T(G)=G$ implies $G^{\prime} \subseteq\left\langle x^{p} \mid x \in G\right\rangle$. That is, the Sylow subgroups of $G$ are powerful p-groups.

For proving the converse, we assume that $G$ is a direct product of powerful $p$-groups of odd order. Let $N \unlhd G$ such that $G / N$ is t-complete. Then $G / N$ is a $p$-group by item (6) of Theorem 3. As quotient of a powerful $p$-group, $G / N$ is powerful. By Corollary 11 the only tcomplete powerful groups are elementary abelian 2-groups. Since $p$ is odd, $G / N$ is trivial. Hence (4) is proved.

For (5) let

$$
\mathcal{M}=\{N \unlhd G \mid G / N \text { is a maximal t-complete quotient of } G\} .
$$

Then $T(G)=\bigcap \mathcal{M}$. If $|\mathcal{M}|=1$, then $G / T(G)$ is t-complete. Now assume we have distinct elements $M, N \in \mathcal{M}$. Since $G /(M \cap N)$ is not t-complete, we have $M N=G$ by Theorem 25 . Thus $G /(M \cap N) \cong$ $G / M \times G / N$. By Theorem 20 the factors $G / M$ and $G / N$ are coprime torsion groups. Now

$$
h: G \rightarrow \bigotimes_{N \in \mathcal{M}} G / N, x \mapsto(x N)_{N \in \mathcal{M}}
$$

is a homomorphism with kernel $T(G)$. Since the projection on each coordinate, $G \rightarrow G / N, x \mapsto x N$, is onto, (5) is proved.

4.9. Maximal t-complete subgroups. We close Section 4 by investigating the subgroup generated by all t-complete subgroups. We take the following easy consequence of Propositions 15 and 16 as motivation.

Proposition 42. Let $G$ be a group of finite exponent d. Let $D_{2 d}$ denote the dihedral group with $2 d$ elements. Let $p$ be a prime not dividing $d$. Then

(1) the group $G \times \mathbb{Z}_{p}$ is not $t$-complete,

(2) the group $G \times D_{2 d}$ is t-complete,

(3) neither t-completeness nor not t-completeness is hereditary to subgroups, to normal subgroups, or to factor groups.

Proof. Item (1) follows from Proposition 15. Item (2) follows from Proposition 16. Item (3) follows from items (1) and (2).

Let $t(G)$ be the subgroup in $G$ which is generated by all t-complete subgroups of $G$,

$$
t(G)=\langle H \leq G| H \text { is t-complete }\rangle .
$$

We summarize our observations about $t(G)$ in Theorem 43 .

Theorem 43. For every group $G$ we have the following: 
(1) Every t-complete subgroup of $G$ is contained in a maximal $t$ complete subgroup; further every maximal t-complete subgroup of $G$ is characteristic in $G$.

(2) $t(G)$ is characteristic in $G$.

(3) If $G$ is t-complete, then $t(G)=G$.

(4) If $G$ is finite and $t(G)=1$, then $|G|$ is odd and any two elements of prime order commute in $G$.

(5) $t(G)$ is either $t$-complete or a direct product of maximal $t$ complete, pairwise coprime torsion subgroups.

Proof. For (1) let $\mathcal{C}$ be a set of t-complete subgroups of $G$ such that $\mathcal{C}$ is linearly ordered by inclusion. We claim that

$$
K=\bigcup \mathcal{C} \text { is t-complete. }
$$

Let $f$ be compatible on $K$ with $f(1)=1$. Then $f$ restricts to a compatible function on every $H \in \mathcal{C}$. Hence either $f(H) \subseteq 1$ for all $H \in \mathcal{C}$ or $\left.f\right|_{H}=\operatorname{id}_{H}$ for all $H \in \mathcal{C}$. In the first case we obtain $f(K)=1$, in the second $f=\mathrm{id}_{K}$. Thus we have (4.21), and the first part of (1) follows.

Next let $H$ be a maximal t-complete subgroup of $G$, and let $\alpha$ be an automorphism of $G$. Then $\langle H, \alpha(H)\rangle$ is t-complete by Theorem 20 . Thus $\alpha(H) \subseteq H$ by the maximality of $H$. Hence $H$ is characteristic.

Items (2) and (3) are immediate from the definition of $t(G)$.

For (4) assume that $G$ is finite and $t(G)=1$. Every subgroup of size 2 is t-complete by Corollary 21. Thus $|G|$ is odd. Further the subgroup of $G$ that is generated by all elements of prime order is abelian by Theorem 38.

Finally for (5), if $H_{1}$ and $H_{2}$ are distinct maximal t-complete subgroups of $G$, then $H_{1}$ and $H_{2}$ are commuting coprime torsion groups by Theorem 20. We conclude that either $t(G)$ is the unique maximal t-complete subgroup of $G$ or that $t(G)$ is the direct product of at least two maximal t-complete subgroups, which are then pairwise coprime torsion groups. This proves (5).

\section{Problems}

Several problems remain open for investigation. By Corollary 4 every finite non-abelian group $G$ is t-complete if its subgroup lattice $\operatorname{Sub}(G)$ satisfies

- $\operatorname{Sub}(G)$ is not a non-trivial direct product, and

- the join of atoms in $\operatorname{Sub}(G)$ is $G$ or the intersection of coatoms in $\operatorname{Sub}(G)$ is 1 . 
For example, for every prime $p$ the lattice $M_{p+1}$, whose $p+1$ elements distinct from 0 and 1 form an antichain, is the subgroup lattice of the t-complete dihedral group $D_{2 p}$ with $2 p$ elements. Moreover, we know that no direct product of two non-trivial lattices and no lattices that are covered by two non-trivial intervals can occur as subgroup lattice of a t-complete group by Theorem 3. This leads to the question:

Problem 1. Which subgroup lattices of finite groups can occur as subgroup lattices of t-complete groups?

For the finite congruence lattice representation problem it would be interesting to consider affine complete $G$-sets in general, not only tcomplete groups.

Problem 2. Which finite (order polynomially complete) lattices can occur as congruence lattice of an affine complete $G$-set?

In our main results, Theorems 2 and 3, we list conditions that imply t-completeness and conditions that prevent t-completeness. This wide variety of properties seems to indicate that the phenomenon is not easily pinned down. Still we would like to have some necessary and sufficient conditions for a group to be t-complete.

Problem 3. Characterize t-complete groups.

One important subproblem would be the study of t-complete $p$ groups.

Problem 4. Characterize t-complete nilpotent groups.

Finally, we feel that the characteristic subgroups $T(G)$ and $t(G)$ are worth investigating as well.

Problem 5. Characterize properties of $T(G)$ and $t(G)$ and their relations to $G$.

\section{ACKNowledgements}

We are grateful to Erhard Aichinger and Csaba Szabó for discussions on this topic. In particular we want to thank Erhard Aichinger for the experimental data on t-complete groups obtained by his computer programs using GAP [5]. Moreover, Proposition 14 is due to him. We thank the anonymous referee for numerous helpful remarks, in particular for calling our attention to the notion of powerful $p$-groups which led to Corollary 11 and consequently improvements of Theorems 3 and 41. This research was partially supported by the Hungarian National Foundation for Scientific Research grants K67870 and N67867 and by the Portuguese Project ISFL-1-143 of CAUL financed by FCT and FEDER. 


\section{REFERENCES}

[1] M. Aschbacher. On intervals in subgroup lattices of finite groups. J. Amer. Math. Soc., 21(3):809-830, 2008.

[2] R. Baddeley and A. Lucchini. On representing finite lattices as intervals in subgroup lattices of finite groups. J. Algebra, 196(1):1-100, 1997.

[3] J. D. Dixon, M. P. F. du Sautoy, A. Mann, and D. Segal. Analytic pro-p-groups, volume 157 of London Mathematical Society Lecture Note Series. Cambridge University Press, Cambridge, 1991.

[4] W. Feit and J. G. Thompson. Solvability of groups of odd order. Pacific J. Math., 13:775-1029, 1963.

[5] The GAP Group. GAP - Groups, Algorithms, and Programming, Version 4.4.12. 2008, (http://www.gap-system.org).

[6] K. Kaarli. Affine complete abelian groups. Math.Nachr., 107:235-239, 1982.

[7] K. Kaarli and A. F. Pixley. Polynomial completeness in algebraic systems. Chapman \& Hall / CRC, Boca Raton, Florida, 2001.

[8] R. N. McKenzie, G. F. McNulty, and W. F. Taylor. Algebras, lattices, varieties, Volume I. Wadsworth \& Brooks/Cole Advanced Books \& Software, Monterey, California, 1987.

[9] P. P. Pálfy. Unary polynomials in algebras, I. Algebra Universalis, 18:262-273, 1984.

[10] P. P. Pálfy and P. Pudlák. Congruence lattices of finite algebras and intervals in subgroup lattices of finite groups. Algebra Universalis, 11:22-27, 1980.

[11] D. J. S. Robinson. A course in the theory of groups, volume 80 of Graduate Texts in Mathematics. Springer-Verlag, New York, second edition, 1996.

[12] R. Schmidt. Subgroup lattices of groups, volume 14 of de Gruyter Expositions in Mathematics. Walter de Gruyter \& Co., Berlin, 1994.

[13] J. W. Snow. OPC lattices and congruence heredity. Algebra Universalis, 58:59$71,2008$.

[14] M. Suzuki. On the lattice of subgroups of finite groups. Trans. Amer. Math. Soc., 70:345-371, 1951.

[15] G. Zappa. Partitions and other coverings of finite groups. Illinois J. Math., 47(1-2):571-580, 2003. Special issue in honor of Reinhold Baer (1902-1979).

Institute of Mathematics, University of Debrecen, Pf. 12, DebreCEN, 4010, Hungary

E-mail address: ghorvath@math.unideb.hu

Caul, Av. Prof. Gama Pinto 2, 1649-003 Lisboa, Portugal \& Institut für Algebra, JKU Linz, Altenberger Strasse 69, 4040 Linz, Austria

E-mail address: stein@cii.fc.ul.pt

Department of Algebra and Number Theory, Eötvös Loránd University, 1117 Budapest, Pázmány Péter sétány 1/C, Hungary

E-mail address: pongeee@cs.elte.hu 